\title{
OTIMIZAÇÃO DO TEMPO DE INJEÇÃO DOS BANCOS DE VAPOR E SOLVENTE EM RESERVATÓRIO DO NORDESTE BRASILEIRO
}

\author{
D. M. S de B. LIMA ${ }^{1}$, E. A. ARAUJO ${ }^{1}$ e J. L. M. BARILLAS ${ }^{1}$ \\ ${ }^{1}$ Universidade Federal do Rio Grande do Norte, Departamento de Engenharia do Petróleo \\ E-mail para contato: davi_msbl@yahoo.com.br
}

\begin{abstract}
RESUMO - Grande parte dos recursos de petróleo do mundo corresponde a hidrocarbonetos viscosos e pesados. Além das maiores dificuldades para produção, o óleo pesado tem um menor valor de mercado em relação ao óleo leve. Existem previsões econômicas de que, para o ano de 2025, o óleo pesado seja a principal fonte de energia fóssil do mundo. Regionalmente o Rio Grande do Norte conta com grandes reservas de petróleo pesados que ainda precisam ser produzidas. A recuperação de óleo em alguns destes reservatórios, pode se utilizar dos processos térmicos de recuperação avançada de petróleo, principalmente através da injeção de vapor, ou através dos processos miscíveis. O método SAS (Steam Alternating Solvent) procura combinar as vantagens do $S A G D$ e do $V A P E X$ para minimizar o consumo de energia por unidade de óleo recuperada. As configurações dos poços são similar ao do processo $S A G D$, ou seja, um poço injetor horizontal e um poço produtor paralelo situado abaixo dele. Neste estudo foi feita uma análise de sensibilidade dos parâmetros operacionais: tempo de injeção e volume de solvente injetado, através do planejamento estrela, a fim de verificar a influência estatisticamente significativa desses fatores na recuperação de óleo, usando o método SAS. Os resultados do estudo da sensibilidade desses parâmetros mostraram a dependência de todos eles no fator de recuperação final, obtendo um aumento na recuperação do óleo em relação ao modelo sem injeção.
\end{abstract}

\section{INTRODUÇÃO}

O aumento da importância dos óleos pesados dentro do cenário energético viabiliza a exploração de reservatórios considerados inicialmente como não rentáveis. Isso leva à procura de tecnologias que otimizem o processo de desenvolvimento de um campo desse tipo.

Regionalmente, o Rio Grande do Norte conta com grandes reservas de petróleo pesado que ainda precisam ser produzidas. A recuperação de óleo em alguns desses reservatórios pode ser incrementada por meio dos processos térmicos de recuperação avançada de petróleo, principalmente, a injeção de vapor. Não obstante, a aplicação dessa técnica acarreta uso excessivo de água e de energia para gerar o vapor. Um método que estar sendo estudado 
atualmente, indicado para a recuperação de óleos pesados, é a injeção de vapor alternada com solvente (SAS - Steam Alternating Solvent). O processo SAS é composto por um método térmico (injeção de vapor) e um método miscível (injeção de solvente) com a finalidade de causar a redução da viscosidade e das tensões interfaciais, melhorando assim deslocamento do óleo até o poço produtor. A Figura1 mostra uma representação esquemática do processo.

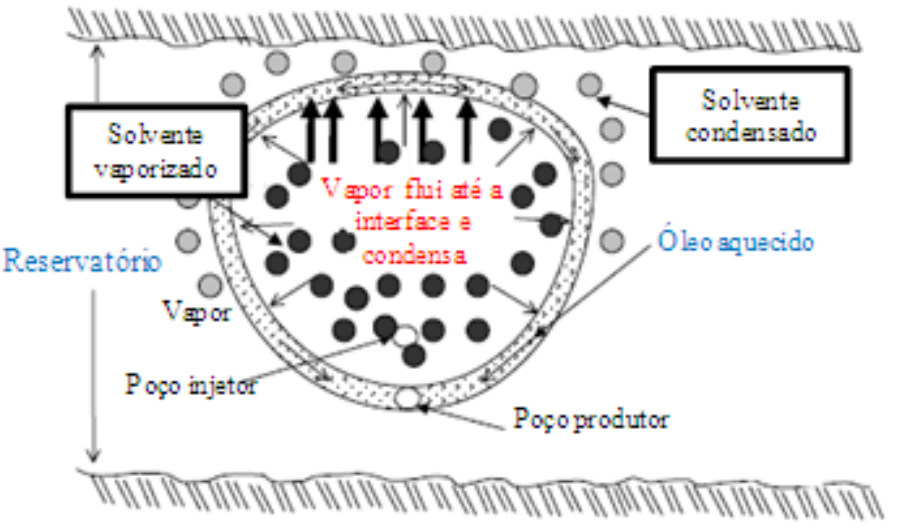

Figura 1 - Esquema do processo SAS

(Fonte: Modificado de Zhao, 2004).

Este processo não tem sido aplicado no Brasil, no entanto, o que torna necessário estudar a sua aplicabilidade às realidades regionais, como forma de provar sua viabilidade. A principal motivação para o desenvolvimento deste estudo é a necessidade de avaliar e adaptar as tecnologias atuais à realidade dos reservatórios brasileiros e mundiais. É importante não apenas explorar e encontrar mais reservatórios de óleo, mas também identificar qual o método mais adequado para um determinado tipo de reservatório para assim produzi-los economicamente e satisfatoriamente. De forma a compreender o mecanismo da injeção alternada de vapor e solvente $(S A S)$ foi proposto um estudo utilizando o simulador numérico STAR 2012.10 da CMG (Computer Modelling Group). Neste trabalho foi analisada a influência de alguns parâmetros operacionais na recuperação de óleo, através do processo $S A S$, usando um reservatório semisintético com características do Nordeste brasileiro. Neste estudo, foram realizadas análises de algumas variáveis operacionais tais como: tempo de injeção e volume de solvente injetado. Para avaliar a influência das variáveis independentes $\mathrm{VC}_{7}$ (volume de solvente) e Tinj (tempo de injeção) - sobre a variável dependente FR (fator de recuperação), elaborou-se um planejamento estrela, através do programa STATISTICA 7, totalizando 10 simulações. 


\section{Metodologia e Modelagem do Reservatório}

O modelo considerado foi um reservatório homogêneo com características do nordeste brasileiro. As dimensões da malha e as principais características do reservatório podem ser observadas na Tabela 1.

Tabela 1 - Dados do modelo de reservatório estudado

\begin{tabular}{c|c}
\hline Propriedades & Valor \\
\hline Comprimento - i (m) & 150 \\
Largura - j (m) & 100 \\
Espessura - k (m) & 26 \\
Profundidade do topo do reservatório (m) & 200 \\
Saturação de água conata (\%) & 39 \\
Saturação inicial de óleo $(\%)$ & 61 \\
Temperatura inicial $\left({ }^{\circ} \mathrm{C}\right)$ & 38 \\
Volume de óleo original - $\left(\mathrm{m}^{3} \mathrm{std}\right)$ & 41.257 \\
\hline
\end{tabular}

Os parâmetros operacionais analisados neste trabalho foram o percentual de solvente injetado e o tempo de injeção de vapor e solvente. A vazão de vapor considerada foi de $60 \mathrm{~m}^{3} / \mathrm{dia}$ e o solvente estudado foi o heptano $\left(\mathrm{C}_{7}\right)$. A Tabela 2 mostra os valores dos parâmetros analisados.

Tabela - 2 Parâmetros analisados

\begin{tabular}{c|c}
\hline Parâmetros & Valores \\
\hline Volume de solvente injetado $\mathrm{VC}_{7}(\mathrm{~m} 3 /$ dia $)$ & $64-66-69-72-73$ \\
Tempo de injeção (dias) & $68-182-456-730-843$
\end{tabular}

Na Figura 2, pode ser observada a distribuição inicial de óleo, através da representação 3D do modelo analisado. A saturação de óleo inicial no topo é de 0,61 . No modelo mostrado, o poço injetor estar localizado a $11 \mathrm{~m}$ do poço produtor. Para ambos os poços, foi determinado um comprimento de $150 \mathrm{~m}$, mesmo tendo em vista que o comprimento deve variar entre $600-1000$ $\mathrm{m}$, conforme a literatura. A escolha do comprimento deve-se à simetria e à homogeneidade do reservatório. Sendo assim, para poços maiores, os resultados podem ser extrapolados. 


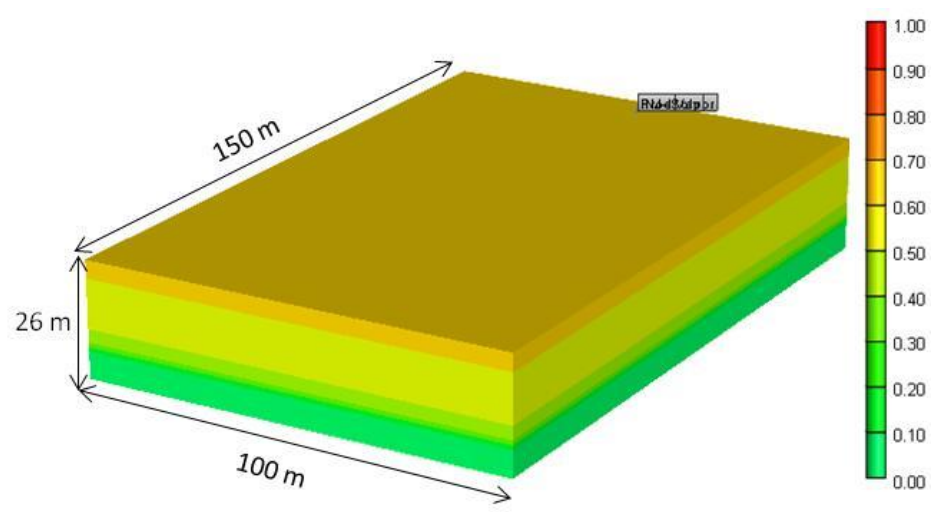

Figura 2 - Modelo do reservatório em 3D (saturação de óleo).

Neste estudo, a modelagem de fluidos foi realizada utilizando um óleo da região do Nordeste do Brasil, ajustado em 9 pseudo-componentes. A Tabela 2 apresenta as composições dos componentes do fluido.

Tabela 2 - Agrupamento e fração mássica inicial

\begin{tabular}{|cc}
\hline Componentes & Mássica inicial (\%) \\
\hline & \\
\hline CO2 & 0.44968 \\
N2 1 - C3 & 0.26981 \\
IC4 - NC5 & 10.35271 \\
C6 - C10 & 0.37973 \\
C11 - C20 & 0.37973 \\
\hline C21 - C30 & 20.25899 \\
C31 - C39 & 27.4629 \\
C40+ & 15.08418 \\
\hline
\end{tabular}

Os intervalos dos parâmetros analisados são apresentados na Tabela 3.

Tabela 3 - Variáveis operacionais para analise de sensibilidade

\begin{tabular}{|c|ccccc|}
\multicolumn{5}{c}{ Nível (Xi) } \\
\hline Parâmetro & $\mathbf{- 1 . 4 1 4 2}$ & $\mathbf{- 1}$ & $\mathbf{0}$ & $\mathbf{1}$ & $\mathbf{1 . 4 1 4 2}$ \\
$\mathrm{VC}_{7}$ (m3/dia) & 64 & 66 & 69 & 72 & 74 \\
Tinj (dias) & 68 & 182 & 456 & 730 & 843.4908 \\
\end{tabular}

Para realizar o planejamento fatorial estrela foram usados os valores correspondentes aos níveis (-1) e (1), a seguir foram calculados os valores centrais e posteriormente os valores para os 
níveis $(-1,41)$ e (1.41), conforme as equações 1 e 2 . A distância vertical entre os poços não teve um aumento signficativo no percentual de óleo recuperado. Por isso, foi mantido a distância entre os poços injetor e produtor de 11 metros.

$X i=($ Nível do fator $-($ Nível alto do fator + Nível baixo do fator $): 2):($ (Nível alto do fator-Nível baixo do fator) : 2)

$$
X=\left(\begin{array}{cll}
i & -i
\end{array}\right): 2
$$

\section{Resultados e discussões}

Neste trabalho, o óleo recuperado foi considerado como a produção acumulada total de óleo menos o solvente produzido em conjunto com o óleo, como mostra a equação 3.

$$
\text { Fr }=\frac{\text { Np total-Np do solvente produzido }}{\text { volume original de oleo }} \times 100
$$

Na Figura 3, pode ser observada a produção acumulada de óleo $(\mathrm{Np})$ em função do tempo para o modelo base (distância entre os poços de $11 \mathrm{~m}, 60 \mathrm{~m}^{3} /$ dia de vapor e $66 \mathrm{~m} 3 /$ dia de solvente injetando $\mathrm{C}_{7}$ ) estudado, isto na versão com e sem injeção de vapor e solvente. Nesta figura se observa que a produção acumulada de óleo é maior quando se injeta vapor e solvente. Foi encontrado que o fator de recuperação em 12 anos do modelo sem injeção de vapor e solvente é de 3,3\% e com injeção alternada é de 69,2\%. O incremento na recuperação do óleo foi de 65,9\% o que pode tornar rentável o uso do processo SAS em reservatórios com condições semelhantes.

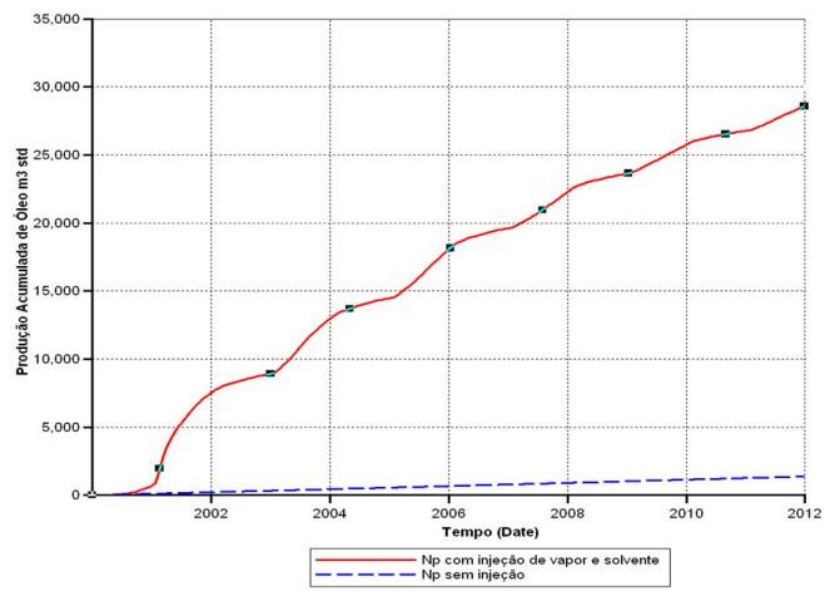

Figura 3 - Produção Acumulada de óleo - Comparação entre o modelo base com e sem injeção de vapor e solvente. 
A Tabela 4 contém os resultados da produção acumulada de óleo e de óleo recuperado em 12 anos para os 10 casos simulados em ordem decrescente da Produção acumulada (Np) e o percentual do Fator de recuperação (FR).

Tabela 4 - Produção acumulada e porcentagem de óleo recuperado em 12 anos de produção

\begin{tabular}{cccc}
\hline $\begin{array}{c}\mathbf{V C}_{\mathbf{7}} \\
\mathbf{M}^{\mathbf{3}} / \mathbf{d i a}\end{array}$ & $\begin{array}{c}\mathbf{T}_{\mathbf{i n j}} \\
(\mathbf{D i a s})\end{array}$ & $\begin{array}{c}\mathbf{N p} \\
(\mathbf{m 3} \text { std) }\end{array}$ & $\begin{array}{c}\mathbf{F r} \\
(\mathbf{\%})\end{array}$ \\
\hline $\mathbf{8}$ & 456 & 28.636 & 69,4 \\
$\mathbf{1 0}$ & 182 & 27.508 & 66,6 \\
$\mathbf{1 0}$ & 730 & 28.577 & 69,2 \\
$\mathbf{1 5}$ & 456 & 28.378 & 68,7 \\
$\mathbf{1 5}$ & 456 & 28.378 & 68,7 \\
$\mathbf{1 5}$ & 843 & 28.039 & 67,9 \\
$\mathbf{1 5}$ & 68 & 27.160 & 65,8 \\
$\mathbf{2 0}$ & 182 & 27.352 & 66,2 \\
$\mathbf{2 0}$ & 730 & 28.378 & 68,7 \\
$\mathbf{2 2}$ & 456 & 28.343 & 68,6 \\
\hline
\end{tabular}

Para análise das significâncias estatísticas das variáveis operacionais foi utilizado o diagrama de Pareto. Os valores da variável $t$ de Student de cada fator cujos retângulos estiverem a direita da linha divisória $(p=0,05)$ são estatisticamente significativos ao nível de $95 \%$ de confiança em relação aos demais. Os efeitos positivos estão associados a um aumento da variável resposta, enquanto os efeitos negativos indicam que um aumento daquele parâmetro reduz a variável resposta considerada. Pode ser observado na Figura 4 (à esquerda), os valores da variável $t$ de Student de cada variável e das interações na recuperação de óleo em 12 anos de projeto.
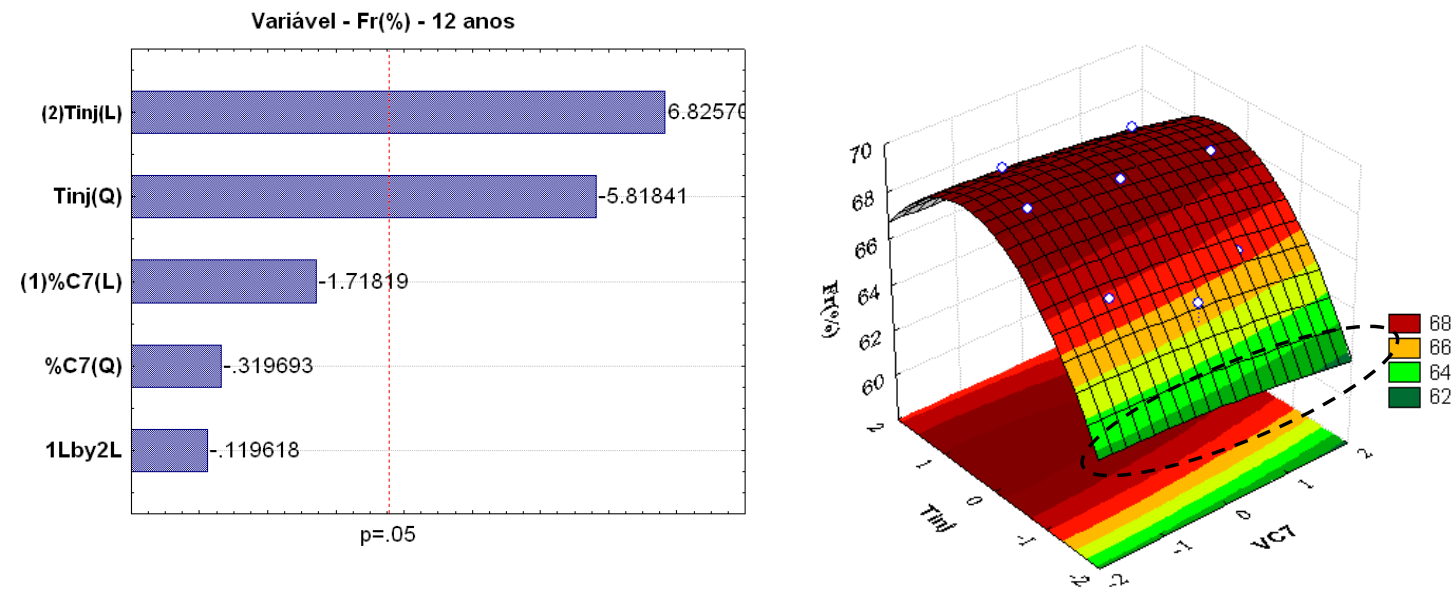

Figura 4 - Diagrama de Pareto dos efeitos padronizados - FR 12 anos de produção/ Superfície de resposta do fator de recuperação em fincão do tempo de injeção e do percentual de solvente injetado. 
Analisando o diagrama de Pareto (Figura 4 à esquerda), percebe-se que o efeito linear do tempo de injeção de vapor e solvente (Tinj) (L), foi o mais significativo, seguido do seu efeito quadrático, Tinj(Q). O percentual de vazão injetado não teve influência estatisticamente significativa sobre a resposta considerada. Ainda na Figura 4, observa-se que o efeito do tempo de injeção de vapor e solvente, Tinj(L), é positivo, ou seja, está associado ao aumento da fração recuperada de óleo. Assim, quanto maior o tempo de injeção de vapor e solvente, maior a recuperação de óleo obtida, considerando os intervalos analisados e as características do reservatório em questão.

A Figura 4 (à direita) mostra superfície de resposta, do tempo de injeção e o volume de solvente injetado no óleo recuperado. Observa-se que a maior fração recuperada de óleo foi obtida para um tempo de injeção de 456 dias (nível 0) combinado com um volume de $64 \mathrm{~m} 3 /$ dia de $\mathrm{C}_{7}$ ( nível 1,4142) atingindo aproximadamente $68 \%$. Observa-se que com o aumento do volume de solvente $\left(\mathrm{VC}_{7}\right)$ injetado não apresentou grande importância sobre a resposta fração recuperada (indicado pelo circulo preto).

A Figura 5 mostra o mapa de viscosidade da fase óleo em um corte transversal do reservatório para o modelo de que injeta $66 \mathrm{~m} 3 /$ dia e $72 \mathrm{~m} 3 /$ dia de heptano $\left(\mathrm{C}_{7}\right)$ alternado a cada 730 dias para quatro períodos de tempo: 2001, 2004, 2008 e 2012. Observa-se que, os quatro períodos de tempo analisados (2001 - 2012), os modelos apresentam comportamentos semelhantes, alcançando a mesma área do reservatório, reduzindo a viscosidade da fase óleo, explicando assim que com o aumento do volume de solvente injetado $\left(\mathrm{C}_{7}\right)$ não há um incremento na fração de óleo recuperada.

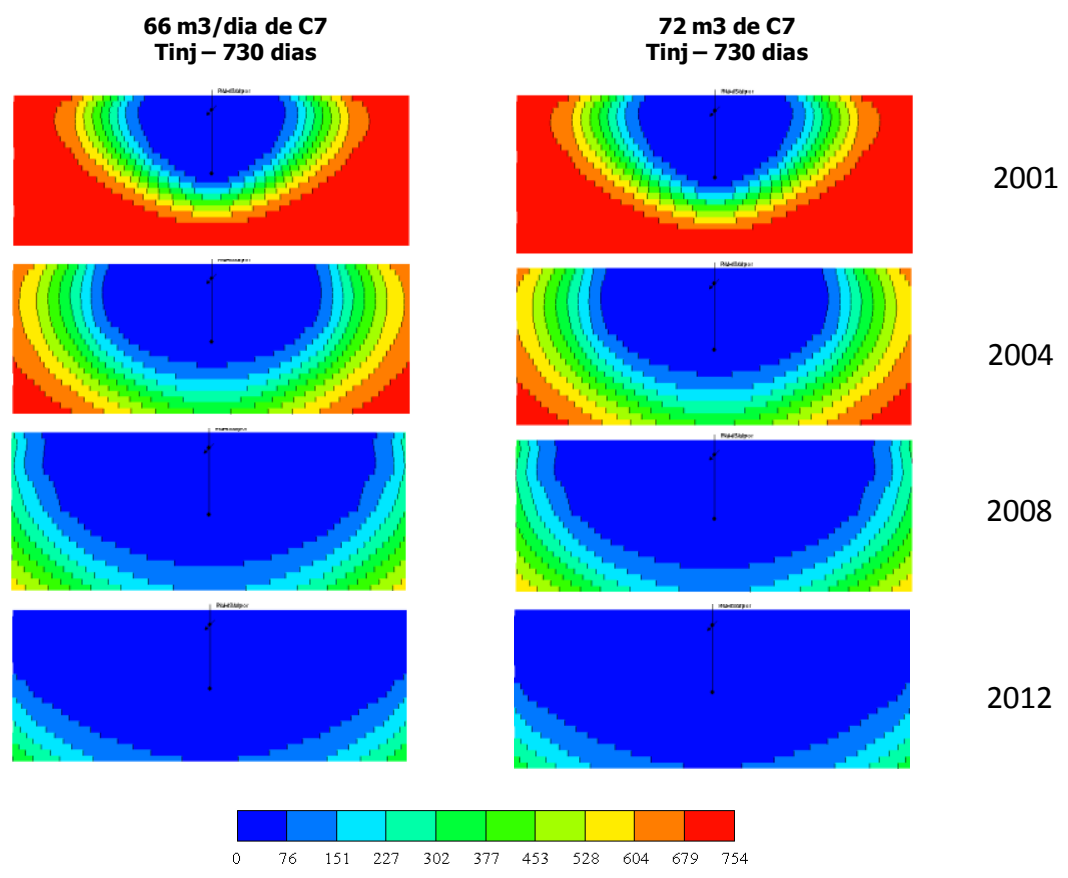

Figura 6 - Viscosidade da fase óleo em 31/12/01; 31/12/04; 31/12/08 e 01/01/12. Injeção de 60 $\mathrm{m}^{3} /$ dia de vapor + Injeção de $66 \mathrm{~m}^{3} /$ dia de solvente e $72 \mathrm{~m}^{3} /$ dia de solvente alternado no período de tempo de 730 dias. 


\section{CONCLUSÕES}

As principais conclusões obtidas deste estudo foram:

Considerando uma viscosidade do óleo de $745 \mathrm{cP}$, o método SAS promoveu um $\Delta \mathrm{Fr}$ em relação a recuperação primária de $65,9 \%$;

Considerando as vazões de injeção analisadas, como aumento do volume de solvente injetado não houve um aumento significativo na produção acumulada de óleo;

A análise de sensibilidade das variáveis operacionais mostrou que para um tempo de injeção alternada (vapor e solvente) de 456 dias combinado com um volume de solvente de $64 \mathrm{~m} 3 /$ dia, proporcionou uma maior recuperação de óleo, atingindo 68\% de óleo recuperado.

\section{AGRADECIMENTOS}

Ao programa PRH -PB221 pelo suporte financeiro, ao LEAP (laboratório de estudos avançados de petróleo), à CMG (Computer Modelling Group) pelo fornecimento dos programas usados.

\section{REFERENCIAS}

ALBOUDWAREJ, H.; FELIX, J.; TAYLOR, S. Highlighting heavy oil, Oilfield Review. p.34-53, Jun. 2006.

BARILLAS, J. L. M. Estudo da recuperação de óleo por drenagem gravitacional assistida por injeção de vapor. Tese de Doutorado, Universidade Federal do Rio Grande do Norte, 165 p., Natal, 2008.

DAS, S. K. Vapex: An efficient process for the recovery of heavy oil and bitumen. SPE 50941. Março, 1998

ROSA, A. J.; CARVALHO, R. S.; XAVIER, J. A. D. Engenharia de reservatórios de petróleo. Rio de Janeiro. Ed. Interciência, 2006;

STARS User's Guide, Advanced process and thermal reservoir simulator, Computer Modelling Group Ltd, 2007.

THOMAS, J. E. "Fundamentos de engenharia de Petróleo", Editora Interciência, Rio de Janeiro, 2001;

ZHAO, L. Steam alternating solvent process. SPE 86957. Março 2004. 